\title{
Clinical Values of miR-23a-3p in Oral Lichen Planus and Its Role in Keratinocyte Proliferation and Inflammatory Response
}

\author{
Jian Wang ${ }^{1, *}$ \\ Mingyan $\mathrm{Hu}^{2, *}$ \\ Leilei $\mathrm{Li}^{2}$ \\ 'Department of Stomatology, Dongying \\ Hospital of Traditional Chinese Medicine, \\ Dongying, Shandong, 257000, People's \\ Republic of China; ${ }^{2}$ Department of \\ Stomatology, Dongying People's Hospital, \\ Dongying, Shandong, 25709I, People's \\ Republic of China
}

*These authors contributed equally to this work
Correspondence: Leilei Li Department of Stomatology, Dongying People's Hospital, No. 317, South Dongcheng Road, Dongying, 25709I, Shandong, People's Republic of China Tel/Fax +86-546-890I 234

Email lileileidongying@।63.com
Purpose: Oral lichen planus (OLP) is a chronic inflammatory disease occurring in the oral cavity, and several miRNAs have been identified to be involved in the disease progression and malignant transformation. This study investigated the expression changes of miR-23a-3p in OLP patients, and further explored its functional role in keratinocyte cell proliferation and inflammatory response.

Patients and Methods: Fifty buccal mucosal tissue samples were collected from OLP patients. HaCaT cells were cultured with lipopolysaccharides (LPS) to mimic the condition of OLP in vitro. RNA extraction and quantitative real-time PCR (qRT-PCR) were used for the measurement of miR-23a-3p levels. The cell viability and inflammation were detected by using cell counting kit-8 (CCK-8) and enzyme-linked immunosorbent assay (ELISA). The target gene of miR-23a-3p was verified by using luciferase reporter assay.

Results: Compared with the control group, miR-23a-3p was significantly downregulated in the buccal mucosal tissues of OLP patients, and a remarkably decreased level of miR-23a-3p was detected in patients with erosive OLP. ROC curve demonstrated the diagnostic value of miR-23a-3p for OLP with the AUC of 0.908 , it can also distinguish erosive OLP from the non-erosive ones. MiR-23a-3p level was negatively associated with RAE (reticular, atrophic, erosive) score in OLP patients $(\mathrm{r}=-0.790, \mathrm{P}<0.001)$. The in vitro experiments indicated that overexpression of miR-23a-3p reversed the promotive effect of LPS on HaCaT cell proliferation and reduced the protein levels of TNF- $\alpha$ and IL-6. The cyclin D1 (CCND1) was a direct target gene of miR-23a-3p, it was overexpressed in OLP cell models.

Conclusion: MiR-23a-3p was at the low expression in OLP patients and showed close association with the disease severity. Overexpression of miR-23a-3p might inhibit keratinocyte proliferation and inflammatory response via targeting CCND1.

Keywords: oral lichen planus, miR-23a-3p, keratinocyte cell, CCND1

\section{Introduction}

Oral lichen planus (OLP) is a chronic inflammatory disease occurring in the oral cavity, affecting approximately $2 \%$ of the population, especially middle-aged women. ${ }^{1}$ OLP has a malignant potency, and according to the World Health Organization, approximately $1.63 \%$ of patients initially diagnosed with OLP can develop into oral squamous carcinomas. ${ }^{2}$ Therefore, the early diagnosis and treatment of OLP are crucial to prevent disease deterioration. However, the etiology and pathogenesis of OLP are still not well understood. Keratinocytes are a major cell type in human skin, which plays an important role in normal skin metabolism. ${ }^{3}$ 
Studies have shown that the degeneration of basal keratinocytes plays an important regulatory role in the development and exacerbation of OLP. ${ }^{4}$

MicroRNAs (miRNAs) are a group of small noncoding RNAs, which regulate the target gene expression through binding to their target messenger RNAs (mRNAs). ${ }^{5}$ Specific miRNAs have been reported to be aberrantly expressed in various human diseases, and are involved in the development and pathogenesis of diseases. ${ }^{6}$ In previous studies, miRNAs are reported to be involved in inflammation, metabolism, and development. ${ }^{7,8}$ In the field of OLP, several miRNAs have been identified to be differentially expressed in OLP patients, and play a role in the disease progression and malignant transformation. ${ }^{9,10}$ For example, miR-4731 is identified to be low expressed in OLP patients, furthermore, the low level of miR-4731 also shows close association with the occurrence and survival outcome of oral squamous cell carcinomas (OSCCs). ${ }^{11}$ In addition, several miRNAs have been detected to be aberrantly expressed in OLP cases, such as miR-155, miR-27b, and so on. ${ }^{12,13}$ MiR-23a-3p is a member of the miR-23a-27a-24-2 cluster, it has been suggested to exert its biological functions in different cancers through regulating cellular activation, cell motility and immune cell infiltration. ${ }^{14,15}$ In a study reported by Chen et al, miR-23a-3p was identified to be downregulated in the tissues of oral squamous cell carcinomas (OSCCs) patients, and overexpression of miR-23a$3 \mathrm{p}$ was suggested to suppress tumor proliferation and invasion of OSCC. ${ }^{16}$ Additionally, the dysregulation of miR-23a-3p was also detected in OLP patients through next-generation sequencing. ${ }^{17}$ However, the functions of miR-23a-3p in OLP remain unclear.

In the present study, the expression changes of miR23a-3p were verified in OLP patients using qRT-PCR, and its clinical role was investigated. Additionally, we further explored the functional role of miR-23a-3p in keratinocyte cells behaviors.

\section{Patients and Methods}

\section{Study Subjects}

This study included 50 OLP patients, who were diagnosed with OLP in Dongying People's Hospital from May 2017 to March 2019. The OLP patients were diagnosed according to the clinicopathologically modified WHO diagnostic criteria of OLP. ${ }^{18}$ The exclusion criteria were as follows: cases with the age below 18 or above 70 years old; pregnant women; patients who suffered from oral lesions adjacent to metal crowns or amalgam fillings; individuals with other detectable oral lesions or systemic diseases, or received treatment 3 months before the sample collection. Patients receiving any medication that can cause lichenoid reactions such as antihypertensive drugs and oral hypoglycemics were also excluded from the study. Besides, another 45 healthy individuals were recruited as a control group, who were detected to have no detectable oral lesions or systemic diseases. Control buccal mucosal tissues were collected during wisdom tooth extraction or orthognathic surgery. The OLP and control groups had no significant difference in age and gender. The clinical characteristics were recorded in Table 1. Before treatment, buccal mucosal tissue samples were collected from each participant.

Table I Clinical Characteristics of the Subjects

\begin{tabular}{|l|c|c|c|}
\hline Variables & $\begin{array}{c}\text { Healthy } \\
\text { Group } \\
(\mathbf{n}=\mathbf{4 5})\end{array}$ & OLP $\mathbf{( n = 5 0 )}$ & $\mathbf{P}$ value \\
\hline Age, years & $49.02 \pm 13.00$ & $48.52 \pm 12.33$ & 0.847 \\
\hline $\begin{array}{l}\text { Gender } \\
\text { Male }\end{array}$ & 11 & 14 & 0.694 \\
Female & 34 & 36 & \\
\hline $\begin{array}{l}\text { Smoking } \\
\text { No } \\
\text { Yes }\end{array}$ & 35 & 38 & 0.837 \\
\hline $\begin{array}{l}\text { Drinking } \\
\text { No }\end{array}$ & 10 & 12 & \\
Yes & 37 & 40 & 0.783 \\
\hline Position & 8 & 10 & \\
Single site & - & 23 & \\
Buccal \\
mucosa
\end{tabular}


The Ethics Committee of Dongying People's Hospital approved all procedures of the present study, and written informed consent was obtained from each individual. This study was conducted in accordance with the Declaration of Helsinki.

\section{Cell Culture and Transfection}

The human immortalized skin keratinocyte cell line, $\mathrm{HaCaT}$, was purchased from American Type Culture Collection (Manassas, VA, USA), and cultured in DMEM supplemented with 10\% FBS (Thermo Fisher Scientific, Waltham, MA, USA) at $37^{\circ} \mathrm{C}$ in a humidified atmosphere containing 5\% $\mathrm{CO} 2$. To mimic the condition of OLP in vitro, $\mathrm{HaCaT}$ cells were cultured with $10 \mu \mathrm{g} / \mathrm{mL}$ of LPS for $48 \mathrm{~h}$ according to the previous evidence. ${ }^{19}$ In brief, $\mathrm{HaCaT}$ cells were plated into a six-well plate at the density of $5 \times 10^{4} /$ well and maintained for $12 \mathrm{~h}$. Then, the cells were cultured for another $48 \mathrm{~h}$ in the absence of LPS $(10 \mu \mathrm{g} / \mathrm{mL})$.

To regulate the expression levels of miR-23a-3p, transfection expression was performed when the cells reached the growth stage. Lipofectamine 2000 (Invitrogen, Carlsbad, CA, USA) was used to transfect miR-23a-3p mimic, miR-23a-3p inhibitor, or the negative control (miR-NC) into cells. After transfection, qRT-PCR was used to evaluate the transfection efficiency.

\section{RNA Extraction and Quantitative Real-Time PCR (qRT-PCR)}

Total RNA was extracted by using TRIzol reagent, then was reversed into complementary cDNA by the miRNA cDNA Synthesis Kit (CWBiotech) according to the manufacturer's instructions. Finally, the expression levels of miR-23a-3p were measured by using miRNA qPCR Assay Kit (CWBiotech) in ABI7300 real-time PCR system (Applied Biosystems; Thermo Fisher Scientific, Inc). The following thermocycling conditions were used for the PCR: initial denaturation at $94^{\circ} \mathrm{C}$ for $2 \mathrm{~min}$, followed by 40 cycles of $94^{\circ} \mathrm{C}$ for $20 \mathrm{sec}, 60^{\circ} \mathrm{C}$ for $34 \mathrm{sec}$. In the reaction, U6 was used as the internal reference gene, and the relative expression of miR-23a-3p was calculated using the $2^{-\Delta \Delta \mathrm{Ct}}$ method. The primer sequences were as follows: miR-23a-3p (5'-CCAGGAACCCCTCCTTACTC $-3^{\prime}$ and 5'-TCTAGGGATGGTCCGAAGGA-3'); U6 (5'CTCGCTTCGGCAGCACATATACT-3' and 5'-ACGCTT CACGAATTTGCGTGTC-3').

\section{CCK-8 Assay}

The change of the cell viability was measured by using (cell counting kit-8) CCK-8 reagent (Dojindo, Japan). Cells were first seeded in 6-well plates with a density of $4 \times 10^{5}$. Before detection, $10 \mu \mathrm{L}$ of reagent was added to the well plate. After incubation in the incubator for $1 \mathrm{~h}$, the absorbance at $490 \mathrm{~nm}$ was detected using a Bio-Rad iMark plate reader (Bio-Rad Laboratories, Inc). The detection was performed every $24 \mathrm{~h}$, and three consecutive tests were performed to assess the relative viability of $\mathrm{HaCaT}$ cells.

\section{Enzyme-Linked Immunosorbent Assay (ELISA)}

The protein levels of inflammatory cytokines were examined by using the ELISA kits (Boster Biotechnology Company, Wuhan, China) according to the manufacturer's protocols.

\section{Luciferase Reporter Assay}

The candidate target gene of miR-23a-3p was predicted by using TargetScan Release 7.0 (http://targetscan.org/), and then was verified through the luciferase reporter assay. The 3 '-untranslated region (UTR) of CCND1 was cloned into the luciferase reporter vector psiCHECK-2 (Promega Corporation) according to the manufacturer's instruction. Briefly, $500 \mathrm{ng}$ of each reporter construct [wild-type (WT) or mutant 3'-UTR of CCND1] and miR-23a-3p mimic, miR$23 a-3 p$ inhibitor or miR-NC were co-transfected into pre-confluent (60-70\%) HaCaT cells using Lipofectamine 2000 (Invitrogen, USA) for $48 \mathrm{~h}$ at $37^{\circ} \mathrm{C}$. The relative luciferase activity was measured by the Dual-Luciferase Reporter System (Promega Corporation, USA) according to the instructions of the manufacturer. Renilla fluorescence activity was identified as the internal reference.

\section{Statistical Analysis}

All data analysis was carried out using the SPSS version 18.0 software (SPSS Inc., Chicago, IL) and GraphPad Prism 5.0 software (GraphPad Software, Inc., USA). The differences between groups were analyzed by Student's $t$-test or one-way ANOVA analysis. Pearson's analysis was used for correlation analysis. The statistical outlier limits were calculated (greater than two standard deviations from the mean), and no significant outliers were detected. The receiver operating characteristic (ROC) curve was used to detect the diagnostic value of miR-23a-3p. The differences were considered significant when $P<0.05$. 


\section{Results}

\section{Clinical Characteristics of the Study Subjects}

As shown in Table 1, there were 11 males and 34 females in the healthy group, with a mean age of $49.02 \pm 13.00$ years old. The mean age of the OLP patients was 48.52 \pm 12.33 years, including 14 males and 36 females. The statistical analysis results indicated that there was no significant difference in age, gender, smoking, and drinking status between the control and OLP groups (all $P>0.05$ ). In addition, the position and clinical classification of OLP patients were recorded in Table 1.

\section{MiR-23a-3p is Downregulated in OLP}

\section{Patients}

The expression levels of miR-23a-3p were detected by qRT-PCR in buccal mucosal tissues of OLP patients. As shown in Figure 1A, miR-23a-3p was significantly lower in OLP patients than that in healthy controls $(P<0.001)$. Additionally, all OLP patients recruited in the present study were divided into the non-erosive group $(\mathrm{n}=29)$ and erosive group $(\mathrm{n}=21)$. It was noted that miR-23a-3p was down-regulated in erosive OLP patients compared with non-erosive cases (Figure 1B, $P<0.001$ ).

\section{Diagnostic Value of miR-23a-3p in OLP Patients}

Considering the dysregulation of miR-23a-3p in OLP patients, a ROC curve was constructed to calculate the diagnostic value of miR-23a-3p for OLP. According to the levels of miR-23a-3p in OLP patients and healthy controls, the ROC curve was constructed. As shown in
Figure 2A, the area under the curve (AUC) was 0.908, with the sensitivity of $84.0 \%$ and specificity of $86.7 \%$ at the cutoff value of 0.820 . It suggested that miR-23a-3p can distinguish OLP patients from healthy controls. Additionally, according to the levels of miR-23a-3p in non-erosive and erosive OLP patients, a ROC curve was also constructed. The results showed that the AUC was 0.831 , with a sensitivity of $71.4 \%$ and specificity of $86.7 \%$ at the cutoff value of 0.550 (Figure 2B). Erosive OLP is featured by more evident oral mucosal damage, it is generally considered to be a more severe form than nonerosive OLP. In terms of the low level of miR-23a-3p in erosive OLP cases, it was concluded that miR-23a-3p might be associated with the severity of OLP.

\section{Correlation of miR-23a-3p with RAE Scores in OLP Patients}

RAE (reticular, atrophic, erosive) score is well known to evaluate the severity of OLP patients. In the current study, we further explored the association of miR-23a-3p level with RAE scores in OLP patients (Figure 3). The results indicated that miR-23a-3p level was negatively associated with RAE score in OLP patients $(\mathrm{r}=-0.790, P<0.001)$, suggesting miR-23a-3p was associated with the severity of OLP.

\section{Overexpression of miR-23a-3p Inhibits $\mathrm{HaCaT}$ Proliferation}

To investigate the role of miR-23a-3p in vitro, HaCaT cells were treated with LPS to mimic the OLP condition. It was found that after LPS treatment, the miR-23a-3p levels were downregulated significantly in $\mathrm{HaCaT}$ cells compared with the control group, which was consistent with the results observed in buccal mucosal tissues of OLP patients (Figure 4A). After cell transfection, the level of
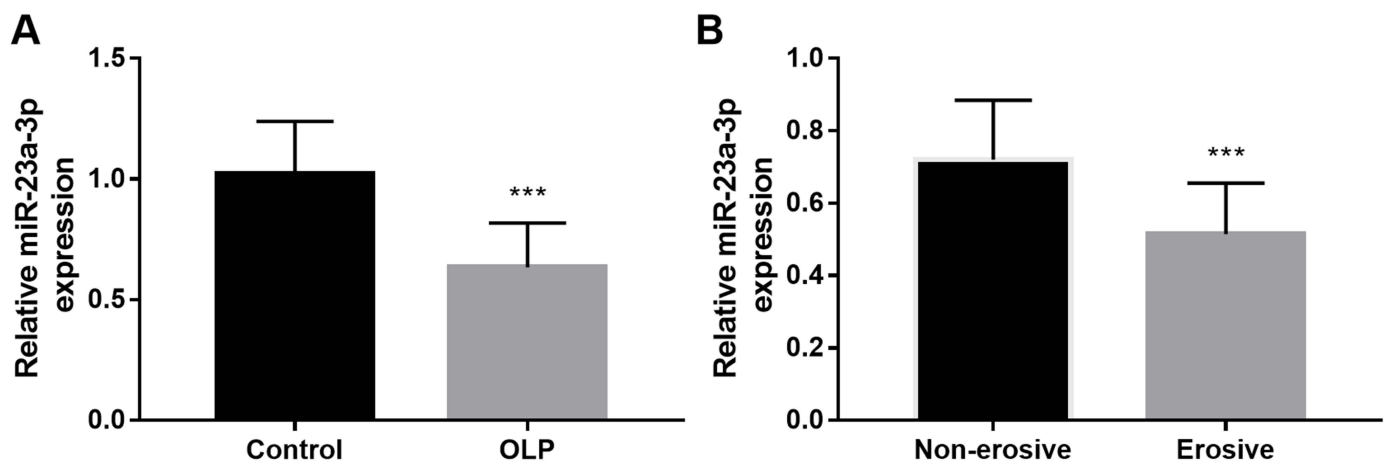

Figure I The expression levels of miR-23a-3p in OLP patients. (A) MiR-23a-3p was significantly lower in OLP patients than that in healthy controls. (B) MiR-23a-3p was down-regulated in erosive OLP patients compared with non-erosive cases. $* * * P<0.001$. 
A

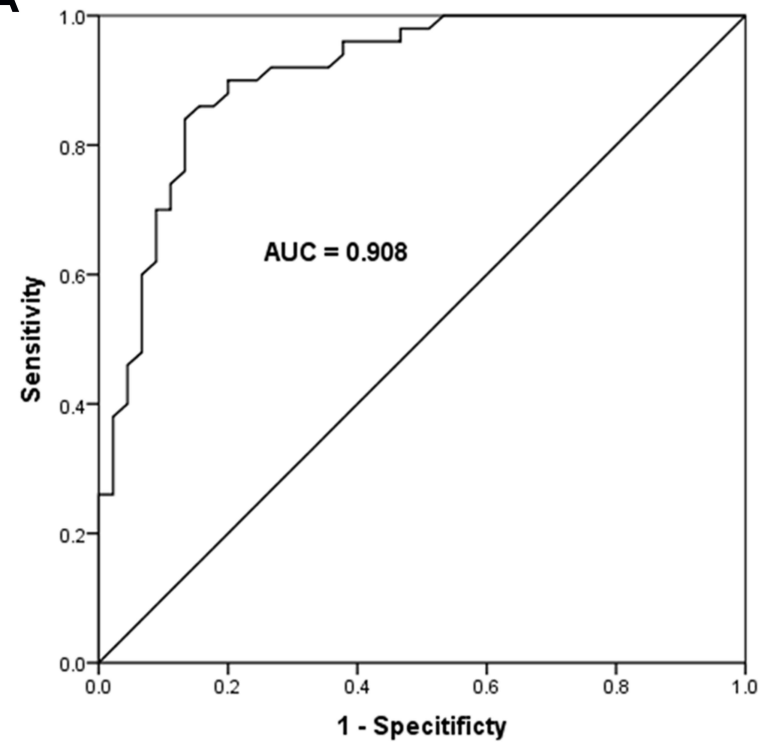

B

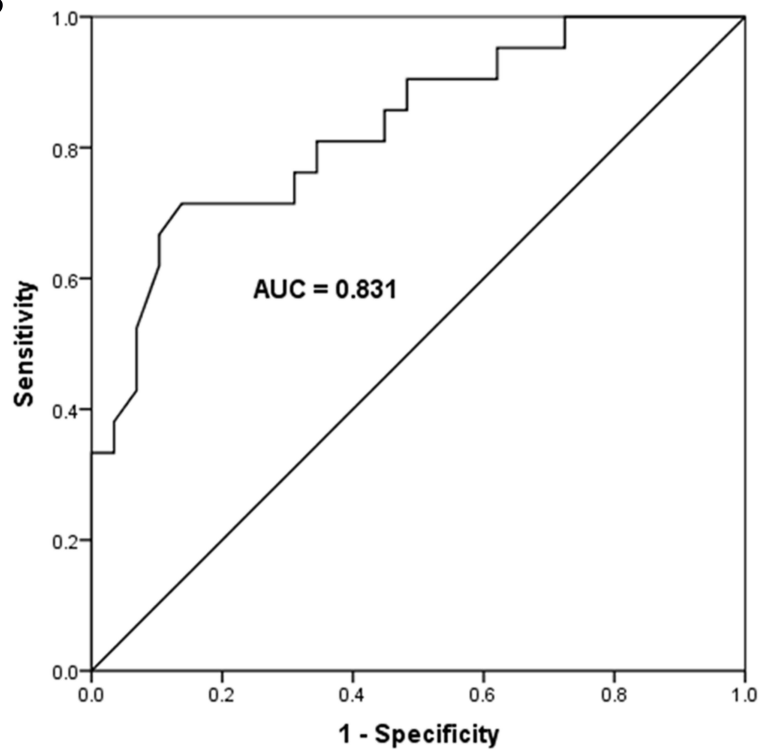

Figure 2 Diagnostic value of miR-23a-3p in OLP patients. (A) According to the levels of miR-23a-3p in OLP patients and healthy controls, miR-23a-3p was suggested to distinguish OLP patients from healthy controls, with the AUC of 0.908 , the sensitivity of $84.0 \%$ and specificity of $86.7 \%$ at the cutoff value of 0.820 . (B) According to the levels of miR-23a-3p in non-erosive and erosive OLP patients, miR-23a-3p could distinguish erosive OLP patients from non-erosive ones, with the AUC of $0.83 \mathrm{I}$, the sensitivity of $71.4 \%$ and specificity of $86.7 \%$ at the cutoff value of 0.550 .

miR-23a-3p was increased significantly by miR-23a-3p mimic transfection, whereas miR-23a-3p inhibitor transfection reduced the level of miR-23a-3p (Figure 4A). The OD value in the CCK-8 assay suggested that LPS treatment inhibited the HaCaT cell viability compared with the untreated control group (Figure 4B). However, overexpression of miR-23a-3p reversed the inhibition of cell viability induced by LPS, whereas miR-23a-3p downregulation further inhibited the cell viability (Figure 4B). Moreover, the protein levels of inflammatory cytokines were further detected. It was found that LPS promoted

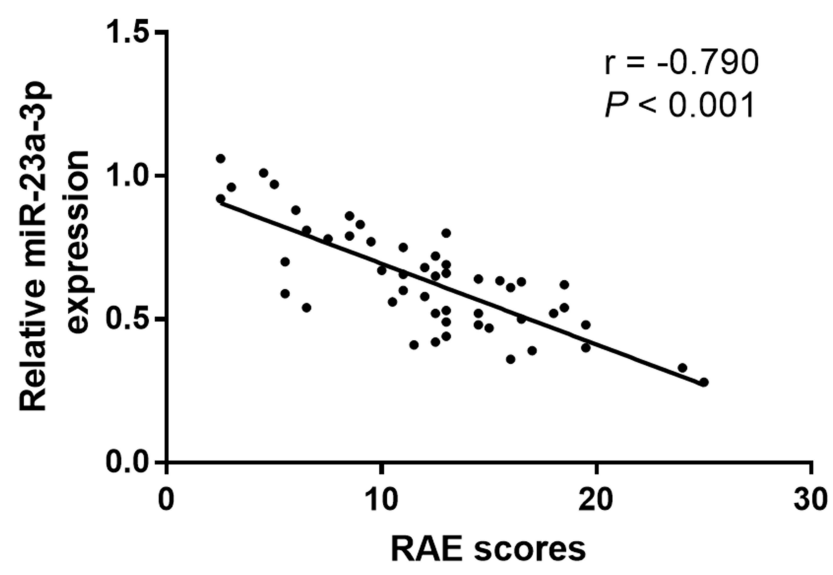

Figure 3 Correlation of miR-23a-3p with RAE scores in OLP patients. MiR-23a-3p level was negatively associated with RAE score in OLP patients $(r=-0.790, P<0.001)$. the release of TNF- $\alpha$ and IL- 6 , which was reversed by miR-23a-3p upregulation. However, downregulation of miR-23a-3p aggravated the release of TNF- $\alpha$ and IL-6 induced by LPS (Figure 4C).

\section{CCNDI is the Target Gene of miR-23a-3p}

According to the TargetScan analysis results, the complementary sequence of miR-23a-3p was found in the 3'-UTR of CCND1 (Figure 5A). Furthermore, the luciferase reporter assay results indicated that overexpression of miR-23a$3 p$ inhibited the luciferase activity of WT 3'-UTR of CCND1 (Figure 5B). But for the luciferase activity of MUT 3'-UTR of CCND1, there was no significant difference among different groups. In addition, CCND1 was upregulated in HaCaT cells after LPS treatment, but miR-23a-3p mimic transfection reduced the level of CCND1, and miR-23a-3p downregulation further increased the level of miR-23a-3p (Figure 5C).

\section{Discussion}

MiRNAs are a class of non-coding RNAs composed of 19-25 nucleotides. MiRNAs can inhibit the target gene expression at the transcriptional levels through binding to the $3^{\prime}$-UTR of mRNA. ${ }^{13}$ The bioinformatic studies have suggested that one-third of known genes may be regulated by different miRNAs. ${ }^{20}$ Many studies have reported that 

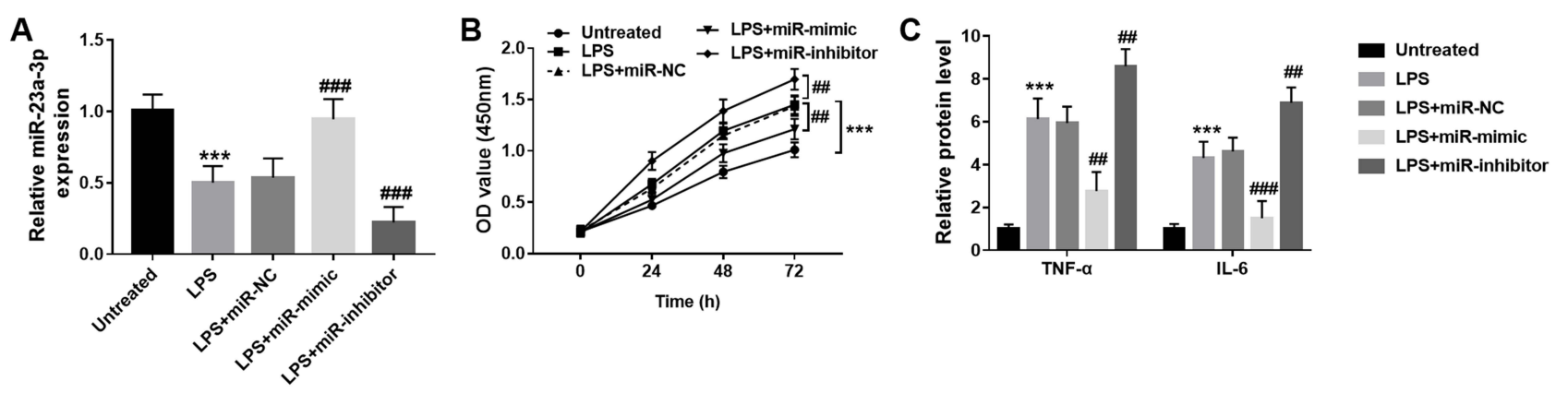

Figure 4 Overexpression of miR-23a-3p inhibits HaCaT proliferation and inflammatory response. (A) The expression level of miR-23a-3p was decreased by LPS treatment, which was reversed by miR-23a-3p mimic transfection. (B) LPS promoted the cell proliferation, which was reversed by miR-23a-3p mimic transfection. (C) LPS promoted the release of TNF- $\alpha$ and IL-6, which was reversed by miR-23a-3p upregulation. However, downregulation of miR-23a-3p aggravated the release of TNF- $\alpha$ and IL-6 induced by LPS. $* * * P<0.001$, compared with untreated group; ${ }^{\# \#} P<0.01,{ }^{\#} P<0.001$, compared with LPS group.

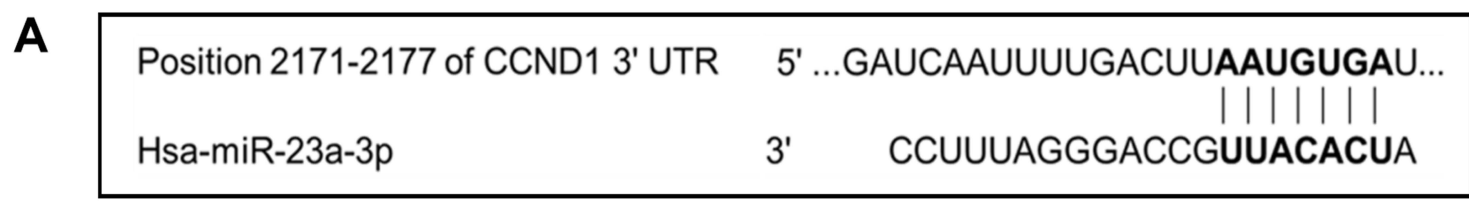

B

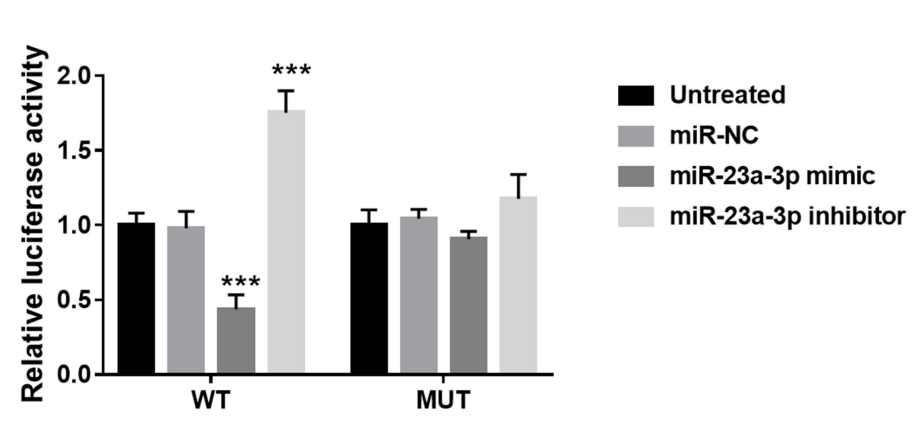

C

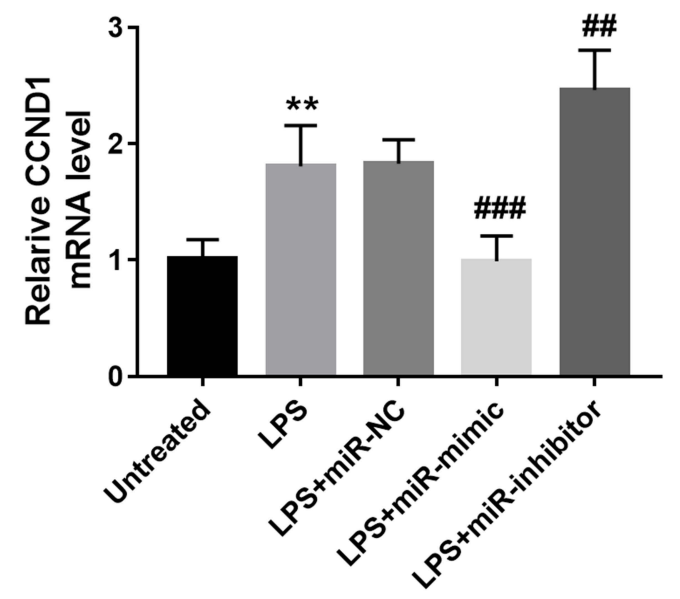

Figure 5 CCNDI was the target gene of miR-23a-3p. (A) According to the TargetScan analysis results, the complementary sequence of miR-23a-3p was found in the 3'-UTR of CCNDI. (B) Overexpression of miR-23a-3p inhibited the luciferase activity of WT 3'-UTR of CCNDI. But for the luciferase activity of MUT 3'-UTR of CCNDI, there was no significant difference among different groups. (C) CCNDI was upregulated in HaCaT cells after LPS treatment, but miR-23a-3p mimic transfection reduced the level of CCNDI, and miR-23a-3p downregulation further increased the level of miR-23a-3p. ${ }^{* * P} P<0.0 \mathrm{I}$, ${ }^{*} * * P<0.00 \mathrm{I}$ compared with untreated group. ${ }^{*} P<0.0 \mathrm{I}$, ${ }^{\prime \prime} P<0.00 \mathrm{I}$, compared with LPS group.

miRNAs are involved in many important cellular processes, such as cell apoptosis, cell differentiation, proliferation, tumorigenesis and tumor inhibition. ${ }^{21,22}$ A large number of researches have proved that miRNA is an important epigenetic regulatory factor in the occurrence and progression of various human diseases. ${ }^{23,24}$

Previous evidence has demonstrated that a variety of miRNAs are differentially expressed in OLP patients. For example, miR-146a and miR-155 were identified to be overexpressed in OLP lesions tissues. ${ }^{25}$ Ma et al reported that approximately $70 \mathrm{miRNAs}$ were identified to be differentially expressed in OLP patients compared with healthy controls through microarray analysis. ${ }^{26}$ In the present study, we confirmed that miR-23a-3p was down-regulated in the buccal mucosal tissues of OLP patients. And the ROC curve analysis results further indicated that miR-23a-3p expression can distinguish OLP patients from healthy controls. Consistent with the present results, a study by Chen et al reported the downregulation of miR-23a-3p in the oral mucosa of OLP patients by using next-generation sequencing with DESeq and edgeR software algorithms, indicating its potential role in the etiology and pathogenesis of OLP, which supported our present results. ${ }^{17}$ All evidence indicated the potential role of miR$23 a-3 p$ in OLP. 
Oral lichen planus is a chronic disease that can be controlled but not eliminated. An erosive form of OLP results in pain and ulceration, which may most likely transform or develop into malignant tumors. ${ }^{27}$ In the current study, a total of 50 OLP patients were recruited, who were further divided into non-erosive group and erosive group. Then the qRT-PCR results indicated that miR-23a-3p was down-regulated in erosive OLP patients compared with non-erosive cases. Considering the expression changes of miR-23a-3p between erosive and non-erosive OLP patients, we speculated that miR$23 a-3 p$ might be associated with the severity of OLP. RAE score is well known to evaluate the severity of OLP patients. In the current study, we further explored the association of miR23a-3p level with RAE scores in OLP patients, and a significantly negative association was detected between miR-23a-3p level and RAE scores. Additionally, according to the levels of miR-23a-3p in non-erosive and erosive OLP patients, a ROC curve was also constructed. The results indicated that miR-23a-3p can distinguish erosive OLP patients from non-erosive OLP patients. Erosive OLP is featured by more evident oral mucosal damage, it is generally considered to be a more severe form than non-erosive OLP. In terms of the low level of miR-23a-3p in erosive OLP cases, we presented that miR-23a-3p might be associated with the severity of OLP.

OLP is an immune disease, and the pathological process of OLP has been reported to be closely linked to basal keratinocytes degeneration. ${ }^{28} \mathrm{HaCaT}$ cells are a type of immortalized human skin keratinocytes, which are easily grown and passaged, thus $\mathrm{HaCaT}$ cells are suitable for the research of oral keratinocytes. Gram-negative bacterial lipopolysaccharide (LPS) has been widely used in previous studies to induce inflammatory mediators. ${ }^{29}$ Accordingly, in the present study, $\mathrm{HaCaT}$ cells were treated with LPS to establish the in vitro inflammatory OLP model. Initially, we found that after LPS treatment, the miR-23a-3p levels were downregulated significantly in $\mathrm{HaCaT}$ cells, which was consistent with the results observed in buccal mucosal tissues of OLP patients. It has been indicated that cell proliferation of keratinocyte was promoted in OLP progression, the same result was also detected in vitro model of OLP, which were consistent with the results observed in LPS-treated HaCaT cells in the current study. ${ }^{30,31}$ Additionally, the gain and loss function experiments' results suggested that overexpression of miR-23a-3p reversed the effect of LPS on HaCaT cell proliferation. We concluded that miR-23a-3p might be involved in the pathogenesis of OLP through inhibiting keratinocyte proliferation.

OLP is an immune disease, and the pathological process of OLP has been reported to be closely linked to the release of inflammatory cytokines. ${ }^{28}$ In the OLP cell model, LPS promoted the release of TNF- $\alpha$ and IL-6, which was consistent with the previous evidence. Furthermore, the gain and loss function experiment results demonstrated that overexpression of miR-23a-3p inhibited the inflammatory response of keratinocyte cells. miR-23a-3p has been widely reported to be involved in the inflammatory response in various cell types and diseases. In the study of traumatic brain injury, miR-23a$3 p$ is reported to alleviate the brain injury via inhibiting neuroinflammation. ${ }^{32}$ Downregulation of miR-23a-3p is also identified in psoriatic arthritis patients and enhanced secretion of inflammatory cytokines in synovial fibroblasts. ${ }^{33}$ The present results indicated that overexpression of miR-23a-3p inhibited the keratinocyte cell inflammatory response during the progression of OLP, which was supported by the previous evidence.

The cyclin D1 (CCND1) gene, located on chromosome $11 \mathrm{q} 13$, is a proto-oncogenic positive regulator of the cell cycle, driving cells from G1 into S phase checkpoint. ${ }^{34}$ Previous studies have indicated that the expression of cell cycle regulatory proteins is dysregulated in oral premalignant and malignant lesions. ${ }^{34,35}$ In the present study, the luciferase reporter assay results indicated that CCND1 was a direct target gene of miR-23a-3p, and CCND1 was upregulated in the LPS induced OLP cell model. The involvement of CCND1 in OLP has been widely reported in previous studies. ${ }^{36,37}$ In addition, an elevated level of CCND1 is identified by Yao et al $r$ in the epithelial cells of OLP patients by immunohistochemistry, and it was closed associated with the cell apoptosis index of the lymphocytes. ${ }^{36}$ Besides, CCND1 is also detected to be at the high expression in the mucosa samples of OLP patients, and the aberrant expression is involved in the protective role of miR-138 in OLP. ${ }^{38}$ It is known that CCND1 is a target of the WNT signaling pathway, which has been reported to have a significant role in malignant transformation of oral lesions such as OLP, and contribute to the chronic inflammatory disease. ${ }^{39}$ Accordingly, we speculated that miR-23a-3p might inhibit keratinocyte proliferation and inflammatory response via targeting CCND1. However, the underlying mechanism is worthy of exploration. In the future, other studies involving the role of CCND1 as well as WNT signaling in OLP are needed to confirm our speculation.

\section{Conclusion}

In conclusion, the current study suggested that miR-23a-3p was downregulated in OLP patients, and might be associated with the disease severity. Overexpression of miR-23a-3p might inhibit keratinocyte proliferation via targeting CCND1. The 
present results provide a basis for further study of the molecular mechanisms underlying the development and progression of OLP.

\section{Ethics Statement}

The Ethics Committee of Dongying People's Hospital approved all procedures of the present study, and written informed consent was obtained from each individual.

\section{Disclosure}

The authors report no conflicts of interest in this work.

\section{References}

1. Stasio DD, Mosca L, Lucchese A, et al. Salivary mir-27b expression in oral lichen planus patients: a series of cases and a narrative review of literature. Curr Top Med Chem. 2019;19(31):2816-2823. doi:10.2174/1568026619666191121144407

2. Loro LL, Johannessen AC, Vintermyr OK. Decreased expression of bcl-2 in moderate and severe oral epithelia dysplasias. Oral Oncol. 2002;38(7):691-698. doi:10.1016/S1368-8375(02)00002-7

3. Jarocka-Karpowicz I, Biernacki M, Wronski A, Gegotek A, Skrzydlewska E. Cannabidiol effects on phospholipid metabolism in keratinocytes from patients with psoriasis vulgaris. Biomolecules. 2020;10(3):367. doi:10.3390/biom 10030367

4. Ge X, Xie H, Nguyen T, Zhao B, Xu J, Du J. Renin promotes STAT4 phosphorylation to induce IL-17 production in keratinocytes of oral lichen planus. iScience. 2020;23(4):100983. doi:10.1016/j.isci.2020.100983

5. Guo L, Yin M, Wang Y. CREB1, a direct target of miR-122, promotes cell proliferation and invasion in bladder cancer. Oncol Lett. 2018;16(3):3842-3848.

6. Cheng Y, Zhu H, Gao W. MicroRNA-330-3p represses the proliferation and invasion of laryngeal squamous cell carcinoma through downregulation of Tra2 $\beta$-mediated Akt signaling. Mol Cell Probes. 2020;52:101574. doi:10.1016/j.mcp.2020.101574

7. Chen Z, Liu Y, Yang L, Liu P, Zhang Y, Wang X. MiR-149 attenuates endoplasmic reticulum stress-induced inflammation and apoptosis in nonalcoholic fatty liver disease by negatively targeting ATF6 pathway. Immunol Lett. 2020;222:40-48. doi:10.1016/j.imlet.2020.03.003

8. Yuan J, Zhang Y. Sevoflurane reduces inflammatory factor expression, increases viability and inhibits apoptosis of lung cells in acute lung injury by microRNA-34a-3p upregulation and STAT1 downregulation. Chem Biol Interact. 2020;322:109027. doi:10.1016/j.cbi.2020.109027

9. Du J, Gao R, Wang Y, et al. MicroRNA-26a/b have protective roles in oral lichen planus. Cell Death Dis. 2020;11(1):15. doi:10.1038/ s41419-019-2207-8

10. Zhang J, Chen GY, Wang F, Zhou G. MiR-29b interacts with IFN- $\gamma$ and induces DNA hypomethylation in CD4+ T cells of oral lichen planus. Int J Biol Macromol. 2020;147:1248-1254. doi:10.1016/j. ijbiomac.2019.09.252

11. Gholizadeh N, Emami Razavi A, Mohammadpour H, Tavakol F, Sheykhbahaei N. Association of MAPK and its regulatory miRNAs $(603,4301,8485$, and 4731) with the malignant transformation of oral lichen planus. Mol Biol Rep. 2020;47(2):1223-1232. doi:10.1007/s11033-019-05223-6

12. Tao Y, Ai R, Hao Y, et al. Role of miR-155 in immune regulation and its relevance in oral lichen planus. Exp Ther Med. 2019;17 (1):575-586

13. Chen J, Du G, Chang Y, et al. Downregulated miR-27b promotes keratinocyte proliferation by targeting PLK 2 in oral lichen planus. J Oral Pathol Med. 2019;48(4):326-334. doi:10.1111/jop.12826
14. Tian K, Di R, Wang L. MicroRNA-23a enhances migration and invasion through PTEN in osteosarcoma. Cancer Gene Ther. 2015;22(7):351-359. doi:10.1038/cgt.2015.27

15. Jahid S, Sun J, Edwards RA, et al. miR-23a promotes the transition from indolent to invasive colorectal cancer. Cancer Discov. 2012;2 (6):540-553. doi:10.1158/2159-8290.CD-11-0267

16. Chen F, Qi S, Zhang X, Wu J, Yang X, Wang R. miR-23a-3p suppresses cell proliferation in oral squamous cell carcinomas by targeting FGF2 and correlates with a better prognosis: miR-23a-3p inhibits OSCC growth by targeting FGF2. Pathol Res Pract. 2019;215(4):660-667. doi:10.1016/j.prp.2018.12.021

17. Chen J, Du G, Wang Y, Shi L, Mi J, Tang G. Integrative analysis of mRNA and miRNA expression profiles in oral lichen planus: preliminary results. Oral Surg Oral Med Oral Pathol Oral Radiol. 2017;124(4):390-402 e17. doi:10.1016/j.oooo.2017.05.513

18. Zhang J, Wei MH, Lu R, Du GF, Zhou G. Declined hTERT expression of peripheral blood CD4+ T cells in oral lichen planus correlated with clinical parameter. J Oral Pathol Med. 2016;45(7):516-522. doi:10.1111/jop.12399

19. Wang J, Yang L, Wang L, Yang Y, Wang Y. Forkhead box p3 controls progression of oral lichen planus by regulating microRNA-146a. $J$ Cell Biochem. 2018;119(11):8862-8871. doi:10.1002/jcb.27139

20. Bai Y, Yang W, Yang HX, et al. Downregulated miR-195 detected in preeclamptic placenta affects trophoblast cell invasion via modulating ActRIIA expression. PLoS One. 2012;7(6):e38875. doi:10.1371/journal.pone. 0038875

21. Wang Y, Zeng G, Jiang Y. The emerging roles of miR-125b in cancers. Cancer Manag Res. 2020;12:1079-1088. doi:10.2147/ CMAR.S232388

22. Dai Y, Zheng C, Li H. Inhibition of miR-23a-3p promotes osteoblast proliferation and differentiation. J Cell Biochem. 2019. doi:10.1002/ jcb.29497.

23. Yang JX, Xie P, Li YS, Wen T, Yang XC. Osteoclast-derived miR-23a-5p-containing exosomes inhibit osteogenic differentiation by regulating Runx2. Cell Signal. 2020;70:109504. doi:10.1016/j. cellsig.2019.109504

24. Liu C, Li J, Wang W, Zhong X, Xu F, Lu J. miR-206 inhibits liver cancer stem cell expansion by regulating EGFR expression. Cell Cycle. 2020;19(10):1077-1088. doi:10.1080/15384101.202 0.1739808

25. Xue-yi L, Jing-yu HU, Gang ZH. [Detection of miR-155, miR-146a in PBNCs and tissues from patients with oral lichen planus]. Shanghai Kou Qiang Yi Xue. 2015;24(6):729-734. Chinese.

26. Ma H, Wu Y, Yang H, et al. MicroRNAs in oral lichen planus and potential miRNA-mRNA pathogenesis with essential cytokines: a review. Oral Surg Oral Med Oral Pathol Oral Radiol. 2016;122 (2):164-173. doi:10.1016/j.oooo.2016.03.018

27. Shiva A, Zamanian A, Arab S, Boloki M. Immunohistochemical study of p53 expression in patients with erosive and non-erosive oral lichen planus. $J$ Dent. 2018;19(2):118-123.

28. Cao T, Zhang H, Zhou L, et al. In vitro cell culture system optimization of keratinocytes from oral lichen planus (OLP) patients. Oral Dis. 2017;23(2):225-232. doi:10.1111/odi.12599

29. Pei Y, Cui F, Du X, et al. Antioxidative nanofullerol inhibits macrophage activation and development of osteoarthritis in rats. Int J Nanomedicine. 2019;14:4145-4155. doi:10.2147/IJN.S202466

30. Danielsson K, Ebrahimi M, Nylander E, Wahlin YB, Nylander K. Alterations in factors involved in differentiation and barrier function in the epithelium in oral and genital lichen planus. Acta Derm Venereol. 2017;97(2):214-218. doi:10.2340/00015555-2533

31. Wang J, Luo H, Xiao Y, Wang L. miR-125b inhibits keratinocyte proliferation and promotes keratinocyte apoptosis in oral lichen planus by targeting MMP-2 expression through PI3K/Akt/mTOR pathway. Biomed Pharmacother. 2016;80:373-380. doi:10.1016/j. biopha.2016.02.043 
32. Li Z, Xu R, Zhu X, Li Y, Wang Y, Xu W. MicroRNA-23a-3p improves traumatic brain injury through modulating the neurological apoptosis and inflammation response in mice. Cell Cycle. 2020;19 (1):24-38. doi:10.1080/15384101.2019.1691763

33. Wade SM, Trenkmann M, McGarry T, et al. Altered expression of microRNA-23a in psoriatic arthritis modulates synovial fibroblast pro-inflammatory mechanisms via phosphodiesterase 4B. J Autoimmun. 2019;96:86-93. doi:10.1016/j.jaut.2018.08.008

34. Koontongkaew S, Chareonkitkajorn L, Chanvitan A, Leelakriangsak M, Amornphimoltham P. Alterations of p53, pRb, cyclin $\mathrm{D}(1)$ and cdk4 in human oral and pharyngeal squamous cell carcinomas. Oral Oncol. 2000;36(4):334-339. doi:10.1016/S13688375(99)00093-7

35. Poomsawat S, Buajeeb W, Khovidhunkit SO, Punyasingh J. Alteration in the expression of cdk4 and cdk6 proteins in oral cancer and premalignant lesions. J Oral Pathol Med. 2010;39(10):793-799. doi:10.1111/j.1600-0714.2010.00909.x
36. Yao X, Yin C, Shen LJ, Xie SM. [Expressions of NF-kappaBp65, TRAF2, cyclinD1 and their association with cell apoptosis in oral lichen planus]. Nan Fang Yi Ke Da Xue Xue Bao. 2007;27 (11):1657-1660. Chinese.

37. Martin-Ezquerra G, Salgado R, Toll A, et al. Multiple genetic copy number alterations in oral squamous cell carcinoma: study of MYC, TP53, CCDN1, EGFR and ERBB2 status in primary and metastatic tumours. Br J Dermatol. 2010;163(5):1028-1035. doi:10.1111/ j.1365-2133.2010.09947.x

38. Ghallab NA, Kasem RF, El-Ghani A, et al. Gene expression of miRNA-138 and cyclin D1 in oral lichen planus. Egypt Dent J. 2017;63:625-638. doi:10.1007/s00784-017-2091-5

39. Ejaz I, Ghafoor S. WNT signalling pathway in oral lesions. J Pak Med Assoc. 2019;69(11):1687-1692.
Journal of Inflammation Research

\section{Publish your work in this journal}

The Journal of Inflammation Research is an international, peerreviewed open-access journal that welcomes laboratory and clinical findings on the molecular basis, cell biology and pharmacology of inflammation including original research, reviews, symposium reports, hypothesis formation and commentaries on: acute/chronic inflammation; mediators of inflammation; cellular processes; molecular

\section{Dovepress}

mechanisms; pharmacology and novel anti-inflammatory drugs; clinical conditions involving inflammation. The manuscript management system is completely online and includes a very quick and fair peerreview system. Visit http://www.dovepress.com/testimonials.php to read real quotes from published authors. 